\title{
OTR1 Management of the database system cells: development and implementation
}

\author{
Milena M.V.F. Carvalho ${ }^{1}$, André Queiroz da Silveira², Márcia Arissawa ${ }^{1}$ \\ ${ }^{1}$ Laboratório de Tecnologia de Anticorpos Monoclonais, Vice-Diretoria \\ de Desenvolvimento, Bio-Manguinhos, Fiocruz, RJ \\ 2 Seção de Desenvolvimento de Sistemas, Divisão de Tecnologia da \\ Informação, Bio-Manguinhos, Fiocruz, RJ
}

Introduction: The Monoclonal Antibodies Technology Laboratory (LATAM) works in the development and production of antibodies for different purposes, supporting internal development projects, technology transfers and other institutions which have collaboration with Bio-Manguinhos. Since the beginning of its activities, the Laboratory created a database with approximately five hundred clones, totaling more than five thousand cells currently stored, making unfeasible the control through records and spreadsheets that did not provide the reliability for detecting the actions involved in the processes. Based on this fact, it has become imperative to develop a tool that could provide access to information quickly, ensuring the storage of data in a consistent and secure manner, according to the current regulatory requirements.

Objective: Develop a validatable tool to be employed in the management of biological materials, according to the requirements of Good Manufacturing Practices.

Methodology: The first stage of the project consisted in modeling the processes of the Laboratory using the ARIS tool, from which the scope of the system has been set. Based on this model, the prototype was used as the basis for the development of the current system. This system was developed using requirements for engineering with prototyping. It is used in a network environment, allowing multi-user access on Windows platform. Thus, the validation process was started according to ANVISA RDC 17 criteria.

Results: The system showed satisfactory results regarding its features and specifications required by the processes. There was a need for some adjustments to submit the system for approval. As a result, the system currently supports all the details of the processes that gave rise to every cell and the characterization of the antibodies expressed by each strain as well as consumption data and local storage of these cells.

Conclusion: Based on these results, we can consider that the system met the need of developing a tool for tracking and managing the information in order to modify, store or retrieve information, reducing the risks of inconsistencies or problems with data integrity. Their 
development represented a considerable difference to the processes by providing the reliability and traceability necessary for the development and production of biological materials. Because it is a tool whose characteristics are based on the applicability and flexibility, it can be customized based on the needs of laboratories with related activities, providing a greater range of services to other Institutions that can be benefit from this system.

Keywords: Cell Bank, Database, Traceability, Security 